\title{
MINIMAX ESTIMATION OF RANDOM VECTORS
}

V. VAATMANN. JUHUSLIKE VEKTORITE MINIMAKSHINDAMINE

B. ВААТМАНН. МИНИМАКСНОЕ ОЦЕНИВАНИЕ СЛУЧАЙЫХ ВЕКТОРОВ

(Presented by H. Aben)

1. Let $\xi=\left(\xi_{1}, \xi_{2}, \ldots, \xi_{n}\right)^{T}$ be an observable and $\eta=\left(\eta_{1}, \eta_{2}, \ldots, \eta_{m}\right)^{T}$ a non-observable real-valued random vector. Denote by $\mathbf{E}$ the operator of mathematical expectation.

Let

$$
\begin{gathered}
m_{\xi}=\mathbf{E} \xi, \quad m_{\eta}=\mathbf{E} \eta, \quad D_{\xi}=\mathbf{E}\left[\left(\xi-m_{\xi}\right)\left(\xi-m_{\xi}\right)^{T}\right], \\
D_{\eta \xi}=\mathbf{E}\left[\left(\eta-m_{\eta}\right)\left(\xi-m_{\xi}\right)^{T}\right], \quad D_{\eta}=\mathbf{E}\left[\left(\eta-m_{\eta}\right)\left(\eta-m_{\eta}\right)^{T}\right] .
\end{gathered}
$$

Let $R_{m \times n}$ be the space of all $m \times n$-matrices and $R_{m}$ the space of all $m$-vectors with norms $\|A\|=\left[\operatorname{tr}\left(A A^{T}\right)\right]^{1 / 2}$ and $\|a\|=\left(a^{T} a\right)^{1 / 2}$, respectively.

We shall consider the problem of the linear estimation of the vector $\eta$ by $\xi$ so that the mean square error will be minimized. If $m_{\eta}, m_{\xi}, D_{\eta \xi}$ and $D_{\xi}$ are known, the result may be found, e. g. in [ [ $\left.{ }^{1}\right]$.

In the present paper the case is considered when we do not know $m_{\eta}$ and $D_{\eta \xi}$ exactly, but a set $K \subset R_{m \times n}$ and a set $S \subset R_{m}$ are given such that $D_{\eta \xi} \in K, m_{\eta} \in S$. In this case we determine the linear minimax estimate for $\eta$.

Definition. The linear estimate $\hat{\eta}=L_{0} \xi+c_{0}\left(L_{0} \in R_{m \times n}, c_{0} \in R_{m}\right)$ is called the minimax estimate with respect to the sets $K \subset R_{m \times n}, S \subset R_{m}$ if

$$
\begin{aligned}
& \left.\max _{D_{\eta \xi} \notin K, m_{\eta} \in S} \mathrm{E}\left[(L \xi+c-\eta)^{T}(L \xi+c-\eta)\right]\right|_{L=L_{0}, c=c_{0}}= \\
= & \min _{L \in R_{m \times n^{,}}, c \in R_{m}} \max _{D_{\eta \xi} \in K, m_{\eta} \in S} \mathrm{E}\left[(L \xi+c-\eta)^{T}(L \xi+c-\eta)\right] .
\end{aligned}
$$

A set $S_{a} \subset R_{m}$ is called symmetrical with respect to $a \in R_{m}$ if $S_{a}=S+a$, where $S \subset R_{m}$ is a symmetrical set with respect to all co-ordinate axes.

In the following we shall suppose for simplicity that $m_{\xi}=0$.

2. Let us formulate the main result of this paper.

Theorem. Let $K \subset R_{m \times n}$ and $S_{a} \subset R_{m}$ be closed convex bounded sets and let $S_{a}$ be symmetrical with respect to $a \in R_{m}$. Then $\hat{\eta}=L_{0} \xi+c_{0}$ is the linear minimax estimate for $\eta$ if $c_{0}=a$ and $L_{0}=D_{\eta \xi}^{0} D_{\xi}^{+}$, where 


$$
\operatorname{tr}\left(D_{\eta \xi}^{0} D_{\xi}^{+} D_{\xi \eta}^{0}\right)=\min _{D_{\eta \xi} \in K} \operatorname{tr}\left(D_{\eta \xi} D_{\xi}^{+} D_{\xi \eta}\right)
$$

and $D_{\xi}^{+}$denotes the pseudo-inverse of $D_{\xi}$.

$\mathrm{Proof}$. Transform the expression on the right side of (1).

$$
\begin{aligned}
& \min _{L \in R} \min , c \in R_{m} D_{\eta \xi} \in K, m_{\eta} \in S_{a} \\
= & \min _{L \in R_{m \times n}, c \in R_{m}} \max _{D_{\eta \xi} \in K, m_{\eta} \in S_{a}} \operatorname{tr} \mathbf{E}\left[(L \xi+c-\eta)(L \xi+c-\eta)^{T}\right]= \\
= & \min _{L \in R_{m \times n}, D_{\eta \xi} \in K} \max _{D_{\eta}} \operatorname{tr}\left(L D_{\xi} L^{T}-2 D_{\eta \xi} L^{T}+D_{\eta}\right)+ \\
+ & \min _{c \in R_{m}} \max _{m_{\eta} \in S_{a}}\left[\left(c-m_{\eta}\right)^{T}\left(c-m_{\eta}\right)\right] .
\end{aligned}
$$

It is evident that the function on the right side of (2) is convex in $L \in R_{m \times n}$ and concave in $D_{\eta \xi} \in K$. Using Theorem $37.3\left[{ }^{2}\right]$ and the fact that the rank of $D_{\eta_{\xi}}$ cannot be greater than the rank of $D_{\xi}$, we can rewrite (2) as

$$
\begin{aligned}
& \min _{L \in R_{m \times n} D_{\eta \xi} \in K} \max \operatorname{tr}\left(L D_{\xi} L^{T}-2 D_{\eta \xi} L^{T}+D_{\eta}\right)+\min _{c \in R_{m}} \max _{m_{\eta} \in S_{a}}\left[\left(c-m_{\eta}\right)^{T}\left(c-m_{\eta}\right)\right]= \\
& =\max _{D_{\eta \xi} \in K} \min _{L \in R} \operatorname{tr}\left(L D_{\xi} L^{T}-2 D_{\eta \xi} L^{T}+D_{\eta}\right)+\min _{c \in R_{m}} \max _{m_{\eta} \in S_{a}}\left[\left(c-m_{\eta}\right)^{T}\left(c-m_{\eta}\right)\right]= \\
& =\max _{D_{\eta \xi} \in K} \min _{L \in R} \operatorname{tr}\left[D_{\eta}-D_{\eta \xi} D_{\xi}^{+} D_{\xi \eta}+\left(L D_{\xi}-D_{\eta \xi}\right) D_{\xi}^{+}\left(L D_{\xi}-D_{\eta \xi}\right)^{T}\right]+
\end{aligned}
$$

$+\min _{c \in R_{m} m_{\eta} \in S_{a}}\left[\left(c-m_{\eta}\right)^{T}\left(c-m_{\eta}\right)\right]$.

As $D_{\xi}^{+} \geqslant 0$, it follows that the minimax matrix $L_{0}$ is equal to $D_{\eta \bar{\xi}}^{0} D_{\bar{\xi}}^{+}$, where $D_{\eta \bar{\xi}}^{0}$ minimizes $\operatorname{tr}\left(D_{\eta \bar{\xi}} D_{\bar{\xi}}^{+} D_{\bar{\xi}}\right)$ subject to $D_{\eta \bar{\xi}} \in K$. The corresponding minimum exists according to Theorem 2.1.2 [ $\left.{ }^{3}\right]$.

It remains to prove $c_{0}=a$. Using Theorem $32.3^{\left[{ }^{2}\right]}$, we have

$$
\min _{c \in R_{m}} \max _{m_{\eta} \in S_{a}}\left[\left(c-m_{\eta}\right)^{T}\left(c-m_{\eta}\right)\right]=\min _{c \in R_{m}}\left[\left(c-m_{\eta}(c)\right)^{T}\left(c-m_{\eta}(c)\right)\right],
$$

where sgn $\left(m_{\eta}(c)-a\right)=-\operatorname{sgn}(c-a), m_{\eta}(c)$ belongs to the boundary of $S_{a}$ and $\operatorname{sgn}\left(b_{1}, b_{2}, \ldots, b_{m}\right)^{T}=\left(\operatorname{sgn} b_{1}, \operatorname{sgn} b_{2}, \ldots, \operatorname{sgn} b_{m}\right)^{T}$. Therefore $c_{0}=a$. This completes the proof.

Re marks:

1. It follows from the proof that the maximum mean square error of the linear minimax estimate $\hat{\eta}=L_{0} \xi+c_{0}$ is

$$
\begin{gathered}
\max _{D_{\eta \xi} \in K, m_{\eta} \in S_{a}} E\left[\left(L_{0} \xi+c_{0}-\eta\right)^{T}\left(L_{0} \xi+c_{0}-\eta\right)\right]= \\
=\operatorname{tr}\left(D_{\eta}-D_{\eta \xi}^{0} D_{\xi}^{+} D_{\xi \eta \eta}^{0}\right)+\max _{m_{\eta} \in S_{a}}\left[\left(a-m_{\eta}\right)^{T}\left(a-m_{\eta}\right)\right] .
\end{gathered}
$$


2. If $m_{\xi}$ is known and not zero, we estimate $\eta$ by $\xi-m_{\xi}$ and the minimax linear estimate is equal to $D_{\eta \xi} D_{\xi}^{+}\left(\xi-m_{\xi}\right)+a$.

3. If $D_{\eta}$ is known, the Cramer-Rao inequality yields $D_{\eta \xi} \in K_{1}$, where $K_{1}=\left\{A \in R_{m \times n}: A D_{\xi}^{+} A^{T} \leqslant D_{\eta}\right\}$. In this case we can take $K \cap K_{1}$ instead of $K$.

4. If $\eta$ and $\xi$ are two jointly Gaussian random vectors, then the linear minimax estimate for $\eta$ by $\xi$ is also the minimax estimate and equals $\mathbf{E}_{m}(\eta \mid \xi)$, where $\mathbf{E}_{m}$ is the conditional expectation with respect to the least favorable distribution of $\eta$ and $\xi$, i. e.

$$
\begin{gathered}
\min _{g \in G} \max _{D_{\eta \xi} \in K, m_{\eta} \in S_{a}} \mathbf{E}\left[(g(\xi)-\eta)^{T}(g(\xi)-\eta)\right]= \\
=\min _{g \in G} \mathbf{E}_{m}\left[(g(\xi)-\eta)^{T}(g(\xi)-\eta)\right]=\mathbf{E}_{m}\left[\left(L_{0} \xi+c_{0}-\eta\right)^{T}\left(L_{0} \xi+c_{0}-\eta\right)\right],
\end{gathered}
$$

where $G$ is the set of all measurable functions.

3. Now let us consider a simple example to illustrate the results given above.

We consider the problem of the linear estimation of the $m$-vector $\eta$ in the model

$$
\xi=A \eta+\varepsilon,
$$

where $\xi$ is an $n$-vector of observations, $A$ is an $n \times m$-matrix of known elements, $\varepsilon$ is an $n$-vector of the noise, dependent on $\eta$.

Let $m_{\eta}, D_{\eta}, D_{\varepsilon}$ be known and let $m_{\varepsilon}=0\left\|D_{\eta \varepsilon}\right\|^{2} \leqslant c^{2}$, where $c$ is a known scalar such that $c^{2}<\left\|D_{\eta} A^{T}\right\|^{2}$.

We get

$$
\left\|D_{\eta \xi}-D_{\eta} A^{T}\right\|^{2}=\left\|D_{\eta \varepsilon}\right\|^{2} \leqslant c^{2} .
$$

It can be shown that the function $\operatorname{tr}\left(D_{\eta \xi} D_{\xi}^{+} D_{\xi \eta}\right)$ is minimized subject to $\left\|D_{\eta \xi}-D_{\eta} A^{T}\right\|^{2} \leqslant c^{2}$ by

$D_{\eta \xi}^{0}=\left[\operatorname{tr}\left(D_{\eta} A^{T} D_{\xi}^{+} A D_{\eta}\right)\right]^{-1 / 2}\left\{\left[\operatorname{tr}\left(D_{\eta} A^{T} D_{\xi}^{+} A D_{\eta}\right)\right]^{1 / 2}-c\left[\operatorname{tr} D_{\xi}^{+}\right]^{1 / 2}\right\} D_{\eta} A^{T}$

Therefore the linear minimax estimate for $\eta$ by $\xi$ in the model (4) is $\hat{\eta}=D_{\eta \bar{\xi}}^{0} D_{\xi}^{+} \xi+\left(I-D_{\eta \xi}^{0} D_{\xi}^{+} A\right) m_{\eta}$, where $D_{\eta \xi}^{0}$ is determined by $(5), D_{\xi}=$ $=A D_{\eta} A^{T}+D_{\varepsilon}$ and $I$ is the identity matrix.

If $c^{2} \geqslant\left\|D_{\eta} A^{T}\right\|^{2}$, then $D_{\eta \xi}^{0}=0$ and $\hat{\eta}=m_{\eta}$.

\section{REFERENCES}

1. Л и пц е р Р. Ш., Ш и р я в А. Н., Статистика случайных процессов, М., «Наука», 1974

2. Р ок а фелл а р Р., Выпуклый анализ, М., «Мир», 1973.

3. Л о р а н П.-Ж., Аппроксимация и оптимизация, М., «Мир», 1975.

Academy of Sciences of the Estonian SSR, Institute of Cybernetics
Received

June 21, 1979 